\title{
Ueber das Jodantimon; ron
}

\section{R. Brandes und H. W. Böttger.}

Im XIV. Bande 2. R. S. 135 dirser Zeitschrift ist bereits über das Judantimon eine kleine Notiz gegeben, aus welcher erhellet, dafs Jod und Antimon, zusammengerieben, leicht und schon bei gewöhnlicher Temperatur sich verbinden, dafs aber beim Erhitsen die Verbindung sehr rasch unter Entwicklung von Dämpfen und Wïme und mitunter auch ron Licht und explodirend vor sich geht. Werden 125Jod und 14 Antimon gemischt und in ciner Retorte erhitzt, so destillirt das Jodiur in dichten Diimpfen ther, die meist schon im Retortenhalse sich verdichten zu einer dunklen öligen Flüssigkeit, dic rasch su einem rothen metallisch gliinzenden Körper erstarrt. Etwas des Jodürs in einem Kolben erhitst, legt sich an deu Wänden desselben als ein dünner scharlachrother Uebersug an, wie ein spiegrelndrs Moirt.

Der Zweck der nachfolgenden Versuche ist besonders, die Veränderungen keunen sn lernen, welche das Jodantimon durch Einwirkung von Wisser und Alkohol erleidet. Zavor aber haben wir das Jodantimon sellust einer Analyse unterworfen, un zu sehen, ob das auf die bemerkte Weise entstandene mit der dariber gegebenen Formel übereinstimmt.

1 Gran. des feingeriebenon Jodantimons wurde mit verdünnter Chlorwasserstoffäure digerirt. Es entstand eine völlige Auflösung, Welche beim Verdïnnen mit Wasser sich weifs trübte; es wurde dalier so lange nuch Chlorwasserstofiaiure zugesetst, bis die Auflösung wieder erfolgt war, das Antimon daranf aus dernolben durch Schwefelwacerstoffgas gefallt, das entotandenc 
Schwefelantimon scharf getrocknet, und im bedeckteh Porcellantiegel geglühet; es wog 0,351 Grm., die 0,255 Antimon enthalten.

Die Analyse ergiebt also dio Zusammensetzung des Jodantimuns 2u :

$$
\begin{aligned}
& \text { Antimon.....25,5 } \\
& \text { Jod..........74,5 } \\
& 100 .
\end{aligned}
$$

Dieses Resultat stimmt genau mit der Formel über das Jodantimon Sb Jo oder Sbə Jo.' Diese ergiebt nämlich :

$$
\text { Antimon....25,49 }
$$

Einwirkung des Wassers auf das Jodantimon.

Das Wasser äufsert auf das Jodantimon eine zersetzende Wirkung und man findet in einigen Lehrbüchern angegeben, dafs diese bis zur völligen Zersetzung des Jodürs in Antimonoxyd und Jodwasserstoffsäure gehe. Dafs, wenigstens bei. unsern Versuchen, die Zersetzung nicht 80 weit geht, sondern nur bis zu einer gewissen Grenze Statt findet, wird das Folgende ergeben.

Uebergiefst man gepülvertes Jodantimon mit Wagser, so verändert es alsbald seine Farbe, es verwandelt sich in ein blarsgelbes Pulver und es entsteht eine röthlichgelbe an Farbe der Safrantinctur ähnliche Auflösung. Der Rückstand wie die Auflüsung wurden beide einer Analyse unterworfen.

a) Der unlösliche Rückstand.

Der Rückstand, welchen man durch Behandeln von Jodantimon mit ubngefähr 4-8 Gewichtstheilen Wasser erhält, stellt ein gelbes Pulver dar. Erhitzt man dasselbe in einer etwas weiten Glasröhre, so dafs die Luft zutreten kann, so nimmt es erst eine röthere Farbe an, darauf sublimirt Jodantimon, und wenn man jetzt 
stärker erhitzt, wird Jod frei und in der Retorte bleibt ein Rückstand von vielem Antimonoxyd mit etwas Antimonmetall. Wird das Erhitzen dagegen in einer engen Glasröhre vorgenommen, so dafs der Luftzutritt fast gänzlich ausgeschlossen ist, so sublimirt Jodantimon und Antimonmetall bleibt zurück; von Antimonoxyd bemerkt man nur Spuren.

Wird das gelbe Pulver mit einer Auflösung von Weinsteinsäure digerirt, so verschwindet es gänzlich, man erhält eine vollkommene und farblose Auflösung. Wird diese verdunstet, so scheidet sich ein rothes Pulver von Jodantimon ab in einer fast farblosen syrupartigen Flüssigkeit. Wird das Ganze mit Wasser übergossen, so erhält man wieder eine farblose Auflösung. Die Erforschung dieser Verhältnisse behalten wir uns für eine spätere Untersuchung vor.

Das gelbe Pulver scheint durch Behandeln mit blorsem Wasser nicht weiter verändert zu werden, denn wenn dasselbe aufs Neue mit Wasser geschüttelt wird, so nimmt dieses nichts mehr daraus anf, und bleibt farblos und völlig klar.

Behufs der Analyse dieser Verbindung wurde $1 \mathrm{Gr}$. derselben mit Chlorwasserstoffsüure digerirt, die entstandene Auflösung mit Wasser verdünnt, wieder mit Chlorwasserstoffsäure versetzt, bis der Niederschlag aufgelöst war, und dann durch Schwefelwasserstoff das Antimon gefällt. Nach Glïhen des Niederschlags im bedeckten Porcellantiegel wurden 0,89 Grm. Schwefelantimon erhalten, die 0,6477 Antimonmetall anzeigen.

Zur Bestimmung des Jods wurde 1 Grm. des gelben Pulvers mit einer Auflösung von reinem Kali digerirt, das Pulver erschien darauf völlig weifs; die Auflösung warde abfiltrirt, mit Wasser verdünnt, mit Sal- 
petersäure übersättigt und darauf mit salpetersaurem Silberoxyd gefällt und der Niederschlag geglühet, wodurch 0,64 Grm. Jodsilber erhalten wurden, die 0,3476 Jod enthalten.

Hiernach ist das unlösliche gelbe Pulver in $100 \mathrm{zu}$ sammengesetzt aus :

$$
\begin{array}{r}
\text { Antimon ...664,77 } \\
\text { Jod........34,76 } \\
\hline 99,53 .
\end{array}
$$

Auf diese Verbindang pafst die Formel $\mathbf{S b}_{2} \mathbf{J}=$ 2402,65, die in 100 Theilen ergeben mufs :

$$
\begin{aligned}
& \text { Antimon....67,12 } \\
& \text { Jod.........32,88 } \\
& 100 .
\end{aligned}
$$

Das gelbe Pulver ist also ein basisches Jodür, ein Halb-Jodantimon. Die Analyse stimmt zwar mit dem theoretischen Resultat nicht völlig genau überein, doch läfst sich keine andere Formel darauf anwenden, und die erhaltene Differenz hat wohl nur darin ihren Grund, dafs, wie es bei der Darstellung des basischen Jodürs leicht möglich ist, ein kleiner Theil desselben der Einwirkung des Wassers entgangen sein konnte.

b) Die Auflösung.

Wird die Auflösung, die man durch Behandeln des Jodantimons mit Wasser erhalten hat, von dem entstandenen basischen Jodür abfiltrirt und anfs Neue mit Wasser vermischt, so wird sie trübe und setzt ein röthlichgelbes Pulver ab. Erhitzt man sie in einer Retorte, so geht freies Jod in die Vorlage über mit einer röthlich gefärbten wässrigen Flüssiglkeit, im weitern Verlauf der Concentration stölst die rückständige Flüssiglreit in der Retorte weifse sehr reizende Dämpfe aus, das Destillat wird dunkelschwarz, hat einen unangenehmen Geruch and wenn man endlich den Rückstand in der Retorte 
zur Trockne bringt, 60 bekommt man einen röthlichbraunen Körper. Dieser schien uns eine andere Verbindung ron Jod und Antimon zu sein, wefshalb wir eine Analyse desselben anstellten.

0,44 Grm. des Rückstandes wurden mit verdünnter Chlorwasserstoffsäure bis zur Auflösung digerirt, die Auflösung mit Wasser und neuem Säurezusatz behandelt, wie es bei den andern Analysen bereits erwähnt worden ist. Aus der Auflösung wurden durch Sclrwefelwasserstoff 0,17 $\mathrm{Grm}$. Scliwefelantimon erhalten, woraus 0,124 Grm. Antimon resultiren, auf $1 \mathrm{Grm}$. des Rückstandes mithin 0,31. Es würden hiernach 0,69 für Jod uibrig bleiben.

Die untersuchte Verbindung enthält sonach :

$$
\begin{aligned}
& \text { Antimon ....31,00 } \\
& \text { Jod........69,00 } \\
& \hline 100 .
\end{aligned}
$$

Auf diese Verbindung lälst sich die Formel $\mathrm{Sb} J_{2}$ $=2385,95$ anwenden. Nach dieser berechnen sich :

$$
\begin{aligned}
& \text { Antimon . . . . 33, } \\
& \text { Jod.......66,2 }
\end{aligned}
$$

Dafs das Resultat des Versuchs nicht nahe mit dem der Rechnung übereinstimmt, kann nur in der Darstellungsart und der Beschaffenheit der Verbindung selbst liegen. Wir haben einen directen Versuch gemacht über die Bestimmung des Jodgehaltes, wonacỉ wir von 0,5 Grm. der Verbindung 0,350 Grm. Jodsilber erhielten, was milhin 70 Proc. Jod ergeben würde. Die Verbindung ist daher als ein Doppelt-Jodantimon anzusehen.

Die vorstehenden Versuche zeigen, dås die Verbindung von Jod und Antimon, welche durch Behandeln des Jodantimons mit Wasser in die Auflösnng übergeht, durch Verdunsten u, 6. w. nicht unzersetzt isolirt werden 
kann. Um tiber die Zusammensetzung der in der Auflösung befindlichen Verbindung indefs eine nähere Bestimmung zu erhalten, warde Jodantimun mit der sechsfachen Menge Wasser übergossen, und nach einigen Tagen die gelbbraune Flüssigkeit abfiltrirt. Aus $10 \mathrm{Grm}$. dieser Auflösung wurden nach Verdünner mit Wasser, Behazdeln mit Chlorwasserstoffsäure und Schwefelwasserstoffgas 0,08 Grm. Schwefelantimon erhalten, was 0,058 Grm. Antimon entspricht. Andere $10 \mathrm{Grm}$. der Auflösung wurden mit Kali und darauf mit Salpetersäure behandelt, und daraus durch salpetersaures Silberoxyd ein Niederschlag gefällt, der nach dem Glühen 1,64 Grm. wog, also $0,883 \mathrm{Grm}$. Jod anzeigt.

Die in der Auflösung befindliche Verbindung besteht sonach aus:

$$
\begin{gathered}
\text { Antimon....6,17 } \\
\text { Jod........93,83 } \\
100 .
\end{gathered}
$$

Dieses Verhältnifs würde am meisten mit der Formel $\mathrm{Sb} \mathrm{J}_{\mathfrak{i} \text { s }}$ übereinkommen, ob dieselbe aber darauf sich anwenden läfst, oder vielmehr, ob eine solcho Verbindung wirklich existire, ist eine andere Frage. Die Auflösung läfst sich nicht concentriren, ohne dafs sie zersetzt wird, und so ist es möglich, dafs dieselbe ein Gemenge einer gewissen Verbindung mit freiem Jod sein kann.

Wie dem anch sei, eine Verbindung von $S b J_{13}$ $=1265,269$ würde bestehen aus :

$$
\text { Antimon....6,29 }
$$

Dieses stimmt genau mit dem Versuch überein.

Durch einen besondern Versuch fanden wir, dafs bei der Einwirkung von 5-6 Wasser auf 1 Jodantimon 
ohngefähr $\frac{1}{3}$ des gelben basischen Jodürs $\mathrm{Sb}_{2} \mathfrak{J}$ gebildet wird; von 100 Jodantimon wurden nämlich nahe 34 desselben erhalten. 100 Jodantimon $\mathrm{Sb}_{3}$ enthalten nun 25,39 Antimon. Diese Antimonmenge mufste sich wieder finden in 34 des basischen Jodürs $\mathrm{Sb}_{2} \mathrm{~J}$ und in 66 der aufgelösten Verbindung $S b J_{15}$; erstere enthalten 22,78, letztere 4,15 Antimon, zusammen also 26,98, was mit dem eben bemerkten Gehalt in $100 \mathrm{Sb} \mathrm{J}_{3}$ nahe übereinstimmt und für die Richtiglseit dieser Entwicklung spricht. Da nun aber die Auflösung, wie dieses ihr Verhalten beim Erhitzen ergiebt, freies Jod enthält, was auch ihre dunkle Farbe andeutet, so ist die Einwirkung des Wassers wahrscheinlich complicirter. Vielleicht lärst sie sich auf die Weise erklïren: Es liommen $6 \mathrm{Sb}$ $\mathrm{J}_{3}(=6 \mathrm{At}$. Sb $+18 \Lambda \mathrm{t}$. J) zur Zersetzung, so entstehen: $1 \mathrm{Sb}+15 \mathrm{~J}, 5 \mathrm{Sb}+2 \mathrm{~J}+\frac{1}{2} \mathrm{~J}=6 \mathrm{Sb}+$ $18 \mathrm{~J}$, oder $1 \mathrm{Sb}+15_{2}^{\mathrm{I}} \mathrm{J}, 5 \mathrm{Sb}+2 \frac{1}{2} \mathrm{~J}$, oder $\mathrm{Sb}+3 \mathrm{~J}, 11 ! \mathrm{J}$, $5 \mathrm{Sb}+2 \frac{1}{2} \mathrm{~J}$.

Da nach dem obigen Versuch nun 100 Jodantimon durch Behandeln mit Wasser 66 der aufgelösten Verbindung lieferten, so müssen $6 \mathrm{Sb} \mathrm{J}_{3}=19054,20$, wenn sie mit Wasser behandelt werden, 12575,77 der aufgelösten Verbindung geben. Nun hat die Auflösung, wie wir eben entwickelt haben, enthalten $S b J_{15}$, wovon das Gewicht ist 12652,69 , was mit dem Versuch, der auf lreine absolute Genauigkeit Anspruch machen kann, doch nahe übereinstimmt, und die Richtigleit der angegebenen Erklärung beweisen dürfte.

\section{Einwirkung des Alkohols.}

Durch Einwirkung von Alkohol wird das Jodantimon eben so, wie durch Wasser zersetzt. Die Producte, die durch diese Zersetzung gebildet werden, sind indefs nicht ganz dieselben.

Arch. d. Pharm. II. Reihe. XVII. Bds. 3. Hft. 
Es wurde feingepulvertes Jodantimon $\mathrm{Sb} \mathrm{J}_{3}$ mit Alkohol von 80 Proc. übergossen, der Alkohol nahm bald eine hellgelbe Farbe an, wurde nach und nach dunkler, zuletzt dunkelrothgelb und es sonderte sich ein gelber Niederschlag aus, der lange in der Flüssigkeit suspendirt bleibt, filtrirt man diesen $a b$, und lärst die abfiltrirte Flüssigkeit in einem verkorkten Glase stehen, so bildet sich nach einiger Zeit eine neue Menge des Niederschlages, und dieses wiederholt sich noch öfters, wahrscheinlich bis fast alles Antimon aus der Flüssiglieit sich abgeschieden hat. Der Niederschlag, wie die Auflösung, wurden, wie bei dem Versuch mit Wasser, einer Analyse unterworfen.

a) Untersuchung des Niederschlags.

0,5 Grm. des bei der Behandlung des Judantimons nit Alkohol entstehenden unlöslichen Körpers wurden zur Bestimmung des Antimongehalts eben so behandelt, wie bei den früheren Analysen angegeben wurde. Es wurden $0,44 \mathrm{Grm}$. Schwefelantimon erhalten, also auf 1 Grm. 0,88 Grm. Bei der Analyse des durch Wasser entstehenden unlöslichen gelben Körpers wurden von $1 \mathrm{Grm} .0,89 \mathrm{Grm}$. Schwefelantimon erhalten. Dieses zeigt, dafs in beiden Fällen, durch Wasser wie durch Alkohol, dasselbe unlösliche Product gebildet wird, nämlich dafs in beiden Fällen $\mathrm{Sb}_{2} \mathrm{~J}$ entsteht.

b) Untersuchung der Auflösung.

Vird die Auflösung, welche man durch Einwirken von Alkohol auf Jodantimon erhalten hat, destillirt, so gcht eine röthlich gefärbte Flüssigkeit über, nebst vielem freien Jod. Auffallend ist es, dafs das Destillat bei der fortgesetzten Destillation einen eigenthümlichen meerettigartigen scharfen Geruch annimmt, und der 
Inhalt der Retorte entfernt nach Hirschhornül riecht; vielleicht wird dieses durch eine Einwirkung des aufgelüsten Jodantimons auf den Alkohol bewirkt. Beim Verdampfen des Rückstandes der Destillation in einer Porzellanschale im Wasserdampfbade blieb ein bräunlichrother Körper zurück. 0,5 Grm. desselben wurden auf mehrbemerkte Weise zur Bestimmung des Antimons behandelt. Es wurden dadurch 0,23 Grm. Schwefelantimon erhalten, die 0,167 Antimon entsprechen. Diese Verbindung enthielt also 33,4 Proc. Antimon, und ist mithin ebenfalls genau dieselbe, die durch Einwirkung des Wassers unter den analogen Umständen gebildet wird, $\mathrm{Sb} \mathbf{J}_{2}$.

Da die Auflösung, welche durch Einwirken des Jods auf Alkohol entsteht, ebenfalls durch Verdampfen zersetzt wird, und nach und nach durch längeres Stehen ferner sich verändert, so schien es zwar nicht von Belang, dieselbe zu untersuchen, indefs haben wir doch eine Analyse angestellt, wozu die Auflösung unmittelbar nach der Absonderung des basischen Joảïrs verwendet wurde. $10 \mathrm{Grm}$. der Auflösung gaben 0,22 Grm. Schwefelantimon, die 0,16 Grm. Antimon enthalten, und aus andern $10 \mathrm{Grm}$. der Auflösung wurden 1,5 Grm. Jodsilber erhalten, die 0,81 Grm. Jod anzeigen. Dieses würde mithin ergeben :

$$
\begin{gathered}
\text { Antimon. . . 16,49 } \\
\text { Jod........83,51 } \\
100 .
\end{gathered}
$$

Diese Verhältnisse von Jod und Antimon, wenngleich es ungewils ist, ob sie eine wirkliche Verbindung constituiren, würden der Formel $\mathrm{Sb} \mathrm{J}_{5}=4755,20$ entsprechen. Die Verbindung würde nach dieser Formel bestehen aus : 
Antimon $\ldots .16,93$
Jod. ......83,07
100.

Dieses Resultat beweist, dafs die Einwirkung des Alkohols auf das Jodantimon nicht ganz so ist, als die des Wassers, weun auch in beiden Fällen das unlösliche Product, das daraus resultirt, dasselbe ist. Die entstehende Auflösung ist wenigstens anders zusammengesetzt.

Bei einem besondern Versuch ergab sich, dafs durch die Linwirkung des Alkohols auf 100 Jodantimon Sb $\mathrm{J}_{3}$ gegen $2 \tilde{5}$ des basischen Jodürs $\mathrm{Sb}_{2} \mathrm{~J}$ gebildet wurden, also 75 der aufgelösten Verbindung. In der angežbenen Menge beider Verbindungen mülsten demnach 25,39 Jod enthalten sein. Nun enthalten 25 des basischen Jodürs 13,76, die 75 des fünffachen Jodantimons 12,69 Antimon, zusammen 26,45 Antimon, was zwar nicht genau mit der Rechnung zusammentrifft, was indefs bei Versuchen mit einem der Art sich zersetzenden Körper auch selten der Fall sein kann, aber doch dentlich genug eririebt, dafs es sich, wie angeführt wurde, verhält. Die Erscheinungen bei der Destillation dieser Auflösung zeigen, da「s dieselbe Jod im freien Zustande ausgiebt. Es läfst -sich die Zersetzung des Jodantimons mit Alkohol vielleicht auf die Weise erklären : Es kommen $4 \mathrm{Sb} \mathrm{J}_{3}(=4 \mathrm{At} . \mathrm{Sb}+12 \mathrm{At}$. J) zur Zersetzung, so entstehen: $2 \mathrm{Sb}+\mathrm{J}, 2 \mathrm{Sb}+10 \mathrm{~J}+1 \mathrm{~J}=4 \mathrm{Sb}$ $+12 \mathrm{~J}$. Da in dem obigen Versuch 100 Jodantimon, $\mathrm{Sb} J_{3}$, durch Behandeln mit Alkohol 75 der aufgelösten Verbindung lieferten, so müssen $4 \mathrm{Sb} \mathrm{J}_{3}(=4 \mathrm{Sb}+12$ $\mathrm{J}=12702,80$ ) durch die Behandlung mit Alkohol $2 \mathrm{Sb}$ $+11 \mathrm{~J}=9527,18$ liefern, $2 \mathrm{Sb}_{1}$ । ist aber 10299,15, was bei Versuchen dieser Art keine zu grofse Differenz ist, 
indem wahrscheinlich noch kleine Theilchen der Masso der Zersetyung sich entzogen hatten.

\section{Verhalten einer Auflösung von Jodkalium gegen Jod- antimon.}

Da es nach früheren Versuchen schien, dafs das Jodkalium cine gröfsere auflösende Wirkung auf das Jodantimon ausübe, als blofses Wasser, vielleicht damit selbst bestimmte Verbindungen eingehe, so wurden darüber besondere Versuche angestellt, die aber dieser Voraussetzung nicht entsprachen. Es wurde Jodkalium, in Wasser gelöst, mit Jodantimon, Sb $\mathrm{J}_{3}$, in Verbindung gebracht, und einige Zeit damit in Berülurung gelassen. Es billete sich ein Niederschlag und eine braune Auflösung, die letztere wurde filtrirt, und dem Verdunsten überlassen. Es entstanden darin vollkommene Tetraeder, die nach gehörigem Abspülen als reines Jodkalium sich ergaben. Im Uebrigen verhielt sich die Auflüsung wie die mit blofsem Wasser entstandene.

Obwohl jetzt zu vermuthen war, dafs der Niederschlag dieselbe Zusammensetzung haben werde, wie der durch blofses Wasser erhaltene, so haben wir doch auch diesen einer Analyse unterworfen, um jeden $Z$ weifel in dieser Beziehung zu entfernen. $1 \mathrm{Grm}$. des unlöslichen Körpers, eben so analysirt, wie bereits ohen angeführt, gab 0,80 $\mathrm{Grm}$. Schwefelantimon, entsprechend 6,55 Antimon und $2 \mathrm{Grm}$. lieferten 1,27 Jodsilber, die 0,684 Jod enthalten, oder 34,2 Proc. anzeigen. Hieraus ergeben sich für 100:

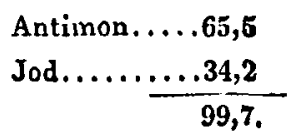


294 Brandes und Böttger: Ueber das Jodantimon.

Es ist auf diese Weise also ebenfalla $\mathrm{Sb}_{2} \mathrm{~J}$ gebildet worden, und keine Verbindung von Jodantimon mit Jodkalium.

\section{Resultate.}

1) Jod und Antimon verbinden sich sehr leicht, schon bei gewöhnlicher Temperatur.

2) Aufser der bekannten und destillirbaren Verbindung $\mathrm{Sb} \mathrm{J}_{3}$ giebt es noch mehre Verbindungen zwischen Jod und Antimon, und entstehen diese durch Zersetzen von Dreifach-Jodantimon durch Wasser oder Weingeist. Die bestimmten Verbindungen dieser Art, welche wir in unsern Versuchen aufgefunden haben, sind :

1) das Halb-Jodantimon, $\mathrm{Sb}_{2} \mathrm{~J}$,

2) das Doppel-Jodantimon, Sb J2.

3) Die Zersetzung des Dreifach-Jodantimons durch Wasser und durch Alkohol ist nicht ganz gleich. Die durch Wasser ist bedeutender, als durch Alkohol. Durch Wasser wird mehr des Halb-Jodantimons, des unlöslichen Niederschlags erzeugt, und daher in die Auflösung eine Verbindung mit mehr Jod übergeführt als beim Alkohol. Die durch Wasser entstandene Auflösung enthält $S_{b} J_{15}$, oder vielleicht $\mathrm{Sb} \mathrm{J}_{2}+13 \mathrm{~J}$, oder $\mathrm{SbJ}_{3}+12 \mathrm{~J}$, oder SbJ $3+$ $11 \frac{1}{2} \mathrm{~J}$, die durch Alkohol entstandene Auflösung aber enthält $\mathrm{Sb} \mathrm{J}_{5}$ oder vielleicht $\mathrm{Sb}_{\mathrm{J}}+3 \mathrm{~J}$, oder $\mathrm{Sb}$ $\mathbf{J} 3+2 \mathrm{~J}$. Die Angabe in mehren Lehrbüchern, dafs Jodantimon durchWasser völlig in Antimonoxyd und Jodwasserstoffsäure zerlegt würde, ist sonach unrichtig; der Niederschlag, wie die Auflösung, enthalten Jod und Antimon.

4) Da Jod und Antimon zu den kräftigsten Arzneimitteln gehören und die Verbindung beider, das 
Geiseler: Ueber Zincum hydrocyanicum. 295

Jodantimon ein leicht und stets in festen Verhältnissen darstellbares Präparat ist, so wäre es gewirs wünschenswerth, wenn die Aerzte dessen therapeutischen Werth in innerlicher, wie in äufserlicher Anwendung prüfen würdea. Wir glauben überzeugt zu sein, dafs dem Arzneischatz dadurch ein kräftiges Mittel zuwachsen wïrde.

\section{Bemerkungen über Zincum hydrocyanicum und Zincum oxydatum; von \\ Dr. Geiseler.}

Jonas wirft im pharmaceutischen Centralblatt 1836, p. 811 die Frage auf, ob unter dem von den Aerzten häufig verschriebenen Zincum hydrocyanicum Cyaneisenzink, eisenblausaures Zinkoxyd (2 $\mathrm{Zn} \mathrm{Cy}_{2}+\mathrm{Fe}$ $\mathrm{C}_{y^{2}}$ ) oder Cyanzink ( $\mathrm{Zn} \mathrm{Cy}_{2}$ ) zu verstehen sei. Folgendes diene als Antwort:

1) Das Zincum hydrocyanicum ist seit dem Jahre 1818 als Arzneimittel in Gebrauch, und die Vorschrift zu dessen Bereitung lautete von $\Lambda$ nfang an dahin, dafs schwefelsaures Zinkoxyd durch blausaures Kali zersetzt werden soll. Nun hat erst im Jahre 1523 Robiquet (Journ. de Pharm. Juillet 1823 und Buchner's Repert. $X V I, 230)$ die Darstellung des reinen blausauren Kalis (Cyankaliums K Cy2), das wahrscheinlich unbekannt war, gelehrt, bis dahin aber war das eisenblausaure Kali (Cyaneisenkalium $2 \mathrm{~K} \mathrm{Cy}_{2}+\mathrm{Fe}_{\mathrm{Cy}_{2}}$ ) nur unter dem Namen blausaures Kali (Kali hydrocyanicum, borussicum, zooticum) bekannt. Da deshalb auch nur dieses zur Dar- 